\title{
Spectroscopic and Theoretical Study of the Intramolecular $\pi$-Type Hydrogen Bonding and Conformations of 3-Cyclopentene-1-amine
}

\author{
Esther J. Ocola and Jaan Laane* \\ Department of Chemistry, Texas A\&M University, College Station, TX 77843-3255, USA \\ Institute for Quantum Science and Engineering, College Station, TX 77843-3255, USA
}

\section{SUPPORTING INFORMATION}

(This file includes two tables and six figures in seven pages.)

\section{Table of Contents}

Page

Table S1. Structural parameters for all of the six conformers of 3CPAM from CCSD/cc-pVTZ computations

Table S2. Observed and calculated vibrational frequencies for the conformers of 3CPAM

Figure S1. Vibrational spectra of the $\mathrm{CH}_{2}$ deformation vibrations ( $v_{4}$ and $v_{17}$ ).

Figure S2. 3CPAM vibrational spectra in the $2800-3100 \mathrm{~cm}^{-1}$ region.

Figure S3. 3CPAM vibrational spectra in the $1180-1450 \mathrm{~cm}^{-1}$ region.

Figure S4. 3CPAM vibrational spectra in the $900-1150 \mathrm{~cm}^{-1}$ region. S6

Figure S5. 3CPAM vibrational spectra in the $630-880 \mathrm{~cm}^{-1}$ region. S7

Figure S6. 3CPAM vibrational spectra in the $185-600 \mathrm{~cm}^{-1}$ region. $\quad$ S7

Corresponding Author, Email address: laane@ chem.tamu.edu, Phone: 979-845-3352 
Table S1. Structural Parameters for all of the Six Conformers of 3CPAM from CCSD/cc-pVTZ computations

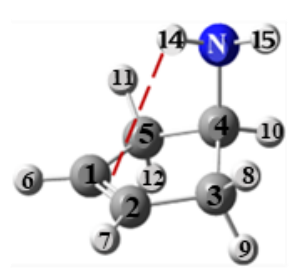

Bond lengths $(\AA)$

$\mathrm{C}_{1}=\mathrm{C}_{2}$

$\mathrm{C}_{1}-\mathrm{C}_{5}$

$\mathrm{C}_{2}-\mathrm{C}_{3}$

$\mathrm{C}_{3}-\mathrm{C}_{4}$

$\mathrm{C}_{4}-\mathrm{C}_{5}$

$\mathrm{C}_{1}-\mathrm{H}_{6}$

$\mathrm{C}_{2}-\mathrm{H}_{7}$

$\mathrm{C}_{3}-\mathrm{H}_{8}$

$\mathrm{C}_{3}-\mathrm{H}_{9}$

$\mathrm{C}_{4}-\mathrm{H}_{10}$

$\mathrm{C}_{5}-\mathrm{H}_{11}$

$\mathrm{C}_{5}-\mathrm{H}_{12}$

$\mathrm{C}_{4}-\mathrm{N}_{13}$

$\mathrm{N}_{13}-\mathrm{H}_{14}$

$\mathrm{N}_{13}-\mathrm{H}_{15}$

\section{Angles (degrees)}

$$
\begin{aligned}
& \mathrm{C}_{1}=\mathrm{C}_{2}-\mathrm{C}_{3} \\
& \mathrm{C}_{5}-\mathrm{C}_{1}=\mathrm{C}_{2} \\
& \mathrm{C}_{2}-\mathrm{C}_{3}-\mathrm{C}_{4} \\
& \mathrm{C}_{4}-\mathrm{C}_{5}-\mathrm{C}_{1} \\
& \mathrm{C}_{3}-\mathrm{C}_{4}-\mathrm{C}_{5} \\
& \mathrm{C}_{3}-\mathrm{C}_{4}-\mathrm{N}_{13} \\
& \mathrm{C}_{5}-\mathrm{C}_{4}-\mathrm{N}_{13} \\
& \mathrm{C}_{1}=\mathrm{C}_{2}-\mathrm{H}_{7} \\
& \mathrm{C}_{1}-\mathrm{C}_{5}-\mathrm{H}_{11} \\
& \mathrm{C}_{1}-\mathrm{C}_{5}-\mathrm{H}_{12} \\
& \mathrm{C}_{2}=\mathrm{C}_{1}-\mathrm{H}_{6} \\
& \mathrm{C}_{2}-\mathrm{C}_{3}-\mathrm{H}_{8} \\
& \mathrm{C}_{2}-\mathrm{C}_{3}-\mathrm{H}_{9} \\
& \mathrm{C}_{3}-\mathrm{C}_{2}-\mathrm{H}_{7} \\
& \mathrm{C}_{3}-\mathrm{C}_{4}-\mathrm{H}_{10} \\
& \mathrm{C}_{4}-\mathrm{C}_{3}-\mathrm{H}_{8} \\
& \mathrm{C}_{4}-\mathrm{C}_{3}-\mathrm{H}_{9} \\
& \mathrm{C}_{4}-\mathrm{C}_{5}-\mathrm{H}_{11}
\end{aligned}
$$

\section{CPAM Conformer}

\begin{tabular}{llllll}
\hline A1 & A2 & B1 & B2 & C & D \\
\hline
\end{tabular}

$\begin{array}{llllll}1.334 & 1.334 & 1.335 & 1.335 & 1.334 & 1.332 \\ 1.511 & 1.510 & 1.509 & 1.510 & 1.510 & 1.508 \\ 1.510 & 1.511 & 1.510 & 1.509 & 1.510 & 1.508 \\ 1.550 & 1.543 & 1.548 & 1.540 & 1.540 & 1.544 \\ 1.543 & 1.550 & 1.540 & 1.548 & 1.540 & 1.544 \\ 1.081 & 1.081 & 1.081 & 1.081 & 1.081 & 1.081 \\ 1.081 & 1.081 & 1.081 & 1.081 & 1.081 & 1.081 \\ 1.092 & 1.090 & 1.096 & 1.096 & 1.094 & 1.092 \\ 1.095 & 1.093 & 1.091 & 1.090 & 1.091 & 1.094 \\ 1.089 & 1.089 & 1.092 & 1.092 & 1.097 & 1.094 \\ 1.090 & 1.092 & 1.096 & 1.096 & 1.094 & 1.092 \\ 1.093 & 1.095 & 1.090 & 1.091 & 1.091 & 1.094 \\ 1.466 & 1.466 & 1.458 & 1.458 & 1.463 & 1.468 \\ 1.014 & 1.013 & 1.013 & 1.012 & 1.013 & 1.013 \\ 1.013 & 1.014 & 1.012 & 1.013 & 1.013 & 1.013\end{array}$

$\begin{array}{llllll}111.3 & 111.5 & 111.3 & 111.6 & 111.5 & 111.8 \\ 111.5 & 111.3 & 111.6 & 111.3 & 111.5 & 111.8 \\ 103.0 & 103.1 & 102.7 & 102.8 & 102.8 & 103.6 \\ 103.1 & 103.0 & 102.8 & 102.7 & 102.8 & 103.6 \\ 104.1 & 104.1 & 104.3 & 104.3 & 104.8 & 105.1 \\ 114.0 & 108.5 & 118.0 & 112.1 & 112.1 & 108.6 \\ 108.5 & 114.0 & 112.1 & 118.0 & 112.1 & 108.6 \\ 125.1 & 125.1 & 125.0 & 125.0 & 125.1 & 125.1 \\ 113.8 & 113.5 & 110.7 & 110.6 & 110.9 & 112.8 \\ 110.4 & 110.3 & 114.0 & 113.6 & 113.3 & 110.8 \\ 125.1 & 125.1 & 125.0 & 125.0 & 125.1 & 125.1 \\ 113.5 & 113.8 & 110.6 & 110.7 & 110.9 & 112.8 \\ 110.3 & 110.4 & 113.6 & 114.0 & 113.3 & 110.8 \\ 123.5 & 123.3 & 123.6 & 123.4 & 123.3 & 123.1 \\ 111.5 & 111.6 & 107.4 & 107.4 & 107.3 & 110.9 \\ 111.4 & 110.5 & 110.4 & 110.4 & 109.8 & 110.9 \\ 111.5 & 111.6 & 112.4 & 112.1 & 112.5 & 111.6 \\ 110.5 & 111.4 & 110.4 & 110.4 & 109.8 & 110.9\end{array}$


Table S1. Continued

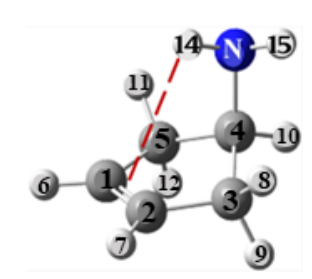

\begin{tabular}{llllll}
\hline A1 & A2 & B1 & B2 & C & D \\
\hline
\end{tabular}

Angles (degrees)

$\mathrm{C}_{4}-\mathrm{C}_{5}-\mathrm{H}_{12}$

$\mathrm{C}_{4}-\mathrm{N}_{13}-\mathrm{H}_{14}$

$\mathrm{C}_{4}-\mathrm{N}_{13}-\mathrm{H}_{15}$

$\mathrm{C}_{5}-\mathrm{C}_{4}-\mathrm{H}_{10}$

$\mathrm{C}_{5}-\mathrm{C}_{1}-\mathrm{H}_{6}$

$\mathrm{H}_{8}-\mathrm{C}_{3}-\mathrm{H}_{9}$

$\mathrm{H}_{10}-\mathrm{C}_{4}-\mathrm{N}_{13}$

$\mathrm{H}_{11}-\mathrm{C}_{5}-\mathrm{H}_{12}$

Angles between bonds

$\mathrm{C}_{1}=\mathrm{C}_{2} / \mathrm{N}_{13}-\mathrm{H}_{14}$

$\mathrm{C}_{1}=\mathrm{C}_{2} / \mathrm{N}_{13}-\mathrm{H}_{15}$

$\mathrm{C}_{1}=\mathrm{C}_{2} / \mathrm{C}_{3}-\mathrm{C}_{4}$

$\mathrm{C}_{2}=\mathrm{C}_{1} / \mathrm{C}_{5}-\mathrm{C}_{4}$

$\mathrm{C}_{3}-\mathrm{C}_{2} / \mathrm{C}_{1}-\mathrm{C}_{5}$

$\mathrm{C}_{2}-\mathrm{C}_{3} / \mathrm{C}_{4}-\mathrm{C}_{5}$

$\mathrm{C}_{5}-\mathrm{C}_{1} / \mathrm{C}_{3}-\mathrm{C}_{4}$

$\mathrm{C}_{3}-\mathrm{C}_{4} / \mathrm{N}_{13}-\mathrm{H}_{14}$

$\mathrm{C}_{3}-\mathrm{C}_{4} / \mathrm{N}_{13}-\mathrm{H}_{15}$

$\mathrm{C}_{5}-\mathrm{C}_{4} / \mathrm{N}_{13}-\mathrm{H}_{14}$

$\mathrm{C}_{5}-\mathrm{C}_{4} / \mathrm{N}_{13}-\mathrm{H}_{15}$

Selected distances (̊̊)

$\mathrm{C}_{1}-\mathrm{H}_{14}$

$\mathrm{C}_{1}-\mathrm{H}_{15}$

$\mathrm{C}_{2}-\mathrm{H}_{14}$

$\mathrm{C}_{2}-\mathrm{H}_{15}$

$\operatorname{Mid}\left(\mathrm{C}_{1}=\mathrm{C}_{2}\right)-\mathrm{H}_{14}$

$\operatorname{Mid}\left(\mathrm{C}_{1}=\mathrm{C}_{2}\right)-\mathrm{H}_{15}$

3CPAM Conformer

$\begin{array}{llllll}111.6 & 111.5 & 112.1 & 112.4 & 112.5 & 111.6 \\ 108.7 & 109.4 & 109.4 & 109.9 & 109.6 & 109.5 \\ 109.4 & 108.7 & 109.9 & 109.4 & 109.6 & 109.5 \\ 111.6 & 111.5 & 107.4 & 107.4 & 107.3 & 110.9 \\ 123.3 & 123.5 & 123.4 & 123.6 & 123.3 & 123.1 \\ 107.1 & 107.5 & 107.1 & 106.9 & 107.6 & 107.3 \\ 107.3 & 107.3 & 107.1 & 107.1 & 112.8 & 112.6 \\ 107.5 & 107.1 & 106.9 & 107.1 & 107.6 & 107.3\end{array}$

$\begin{array}{cccccc}83.5 & -2.9 & 93.2 & -25.2 & -158.9 & -10.8 \\ -177.8 & 85.4 & -159.5 & 87.2 & -26.1 & -172.1 \\ 16.4 & 16.1 & -16.6 & -16.4 & -15.9 & 12.7 \\ -16.1 & -16.4 & 16.4 & 16.6 & 15.9 & -12.7 \\ -0.3 & 0.3 & 0.1 & -0.1 & 0.0 & 0.0 \\ -25.1 & -25.0 & 25.6 & 25.5 & 24.6 & -19.6 \\ 16.5 & 16.7 & -16.9 & -17.0 & -16.3 & 12.9 \\ -56.4 & -174.0 & -55.4 & 178.1 & 63.6 & -179.0 \\ 58.6 & -59.0 & 60.7 & -65.8 & 179.0 & 65.3 \\ 59.0 & -58.6 & 65.8 & -60.7 & -179.0 & -65.3 \\ 174.0 & 56.4 & -178.1 & 55.4 & -63.6 & 179.0\end{array}$

$\begin{array}{llllll}2.906 & 3.690 & 3.830 & 4.165 & 4.351 & 3.714 \\ 3.953 & 2.948 & 4.383 & 3.846 & 4.097 & 3.993 \\ 2.948 & 3.953 & 3.846 & 4.383 & 4.097 & 3.993 \\ 3.690 & 2.906 & 4.164 & 3.830 & 4.351 & 3.714 \\ 2.850 & 3.765 & 3.780 & 4.223 & 4.173 & 3.798 \\ 3.765 & 2.850 & 4.223 & 3.780 & 4.173 & 3.798\end{array}$




\begin{tabular}{|c|c|c|c|c|c|c|c|c|c|c|c|c|c|c|}
\hline \multirow{4}{*}{\multicolumn{2}{|c|}{$\underline{\text { Ring - Pseudo } \mathrm{C}_{2 \mathrm{v}}}$}} & \multicolumn{4}{|c|}{$\mathbf{A}$} & \multicolumn{3}{|r|}{ (3) } & \multicolumn{3}{|c|}{$\mathrm{C}$} & \multicolumn{3}{|c|}{ D } \\
\hline & & \multicolumn{2}{|c|}{ Observed (OB) } & \multicolumn{2}{|c|}{ Calculated } & \multirow{2}{*}{$\begin{array}{c}\mathrm{OB} \\
\Delta \\
\end{array}$} & \multicolumn{2}{|c|}{ Calculated } & \multirow{2}{*}{$\frac{\mathrm{OB}}{\Delta}$} & \multicolumn{2}{|c|}{ Calculated } & \multirow{2}{*}{$\begin{array}{l}\mathrm{OB} \\
\Delta \\
\end{array}$} & \multicolumn{2}{|c|}{ Calculated } \\
\hline & & Freq. $^{\mathrm{a}}$ & $(\mathrm{IR}, \mathrm{R})^{\mathrm{b}}$ & Freq. ${ }^{\mathrm{a}, \mathrm{c}}$ & $(\mathrm{IR}, \mathrm{R})^{\mathrm{b}, \mathrm{c}}$ & & $\Delta^{\mathrm{c}}$ & $(\mathrm{IR}, \mathrm{R})^{\mathrm{b}, \mathrm{c}}$ & & $\Delta^{\mathrm{c}}$ & $(\mathrm{IR}, \mathrm{R})^{\mathrm{b}, \mathrm{c}}$ & & $\Delta^{\mathrm{c}}$ & $(\mathrm{IR}, \mathrm{R})^{\mathrm{b}, \mathrm{c}}$ \\
\hline & & & & & & & & & & & & & & \\
\hline \multicolumn{15}{|c|}{ A1 } \\
\hline$v_{1}$ & $=\mathrm{C}-\mathrm{H}$ stretch & 3072 & $(s, 141)$ & 3075 & $(25,100)$ & 0 & -1 & $(18,85)$ & 8 & 1 & $(16,86)$ & 8 & 1 & $(15,57)$ \\
\hline$v_{2}$ & $\mathrm{CH}_{2}$ symmetric stretch & 2849 & $(s, 67)$ & 2894 & $(43,88)$ & -14 & -15 & $(26,100)$ & 6 & 8 & $(12,100)$ & 13 & 8 & $(42,100)$ \\
\hline$v_{3}$ & $\mathrm{C}=\mathrm{C}$ stretch & 1613 & $(\mathrm{~m}, 88)$ & $1605^{\mathrm{d}}$ & $(28,46)^{d}$ & 4 & $5^{\mathrm{d}}$ & $(13,26)^{b}$ & 4 & $5^{\mathrm{d}}$ & $(10,28)^{b}$ & 16 & 18 & $(3,65)^{\mathrm{d}}$ \\
\hline$v_{4}$ & $\mathrm{CH}_{2}$ deformation & 1452 & $(\mathrm{~m}, 17)$ & 1447 & $(3,77)$ & 3 & 6 & $(2,82)$ & 3 & 9 & $(2,81)$ & -4 & -5 & $(2,99)$ \\
\hline$v_{5}$ & $\mathrm{CH}_{2}$ wag & 1296 & $(w, 10)$ & 1283 & $(1,15)$ & 6 & 14 & $(3,11)$ & -12 & -15 & $(0.4,21)$ & 6 & 9 & $(2,17)$ \\
\hline$v_{6}$ & $=\mathrm{C}-\mathrm{H}$ in plane wag & 1109 & $(w, 58)$ & 1090 & $(0.1,94)$ & 0 & 2 & $(0.4,100)$ & 0 & 0 & $(2,100)$ & 0 & 4 & $(0.2,100)$ \\
\hline$v_{7}$ & Ring stretch & 967 & $(w, 100)$ & 962 & $(1,79)$ & 0 & 3 & $(0.8,76)$ & 0 & 4 & $(0.3,75)$ & 0 & 4 & $(2,91)$ \\
\hline$v_{8}$ & Ring stretch & 804 & $(\mathrm{~s}, 43)$ & 817 & $(62,100)$ & 28 & 8 & $(23,51)$ & 36 & 17 & $(12,53)$ & 31 & 12 & $(5,87)$ \\
\hline$v_{9}$ & Ring angle bend & 735 & $(\mathrm{w}, 9)$ & 728 & $(3,3)$ & -181 & -180 & $(3,27)$ & -161 & -180 & $(0.8,20)$ & -15 & -8 & $(30,4)$ \\
\hline \multicolumn{15}{|l|}{$\mathrm{A} 2$} \\
\hline$v_{10}$ & $\mathrm{CH}_{2}$ antisymmetric stretch & 2929 & $(\mathrm{~s}, 59)$ & 2951 & $(31,48)$ & 10 & 6 & $(27,43)$ & 10 & 8 & $(18,43)$ & 0 & 1 & $(6,33)$ \\
\hline$v_{11}$ & $\mathrm{CH}_{2}$ twist & 1235 & $(w, 6)$ & 1245 & $(10,32)$ & -132 & -135 & $(4,4)$ & -122 & -126 & $(2,3)$ & 15 & 21 & $(4,1)$ \\
\hline$v_{12}$ & $=\mathrm{C}-\mathrm{H}$ out-of-plane wag & 934 & $(\mathrm{~m}, 4)$ & 934 & $(27,4)$ & 7 & 5 & $(22,2)$ & 0 & 0 & $(0.01,3)$ & 0 & -2 & $(0.5,4)$ \\
\hline$v_{13}$ & $\mathrm{CH}_{2}$ rock & 849 & $(\mathrm{~m}, 5)$ & 870 & $(23,9)$ & 10 & 17 & $(11,3)$ & 24 & 21 & $(0.02,2)$ & 5 & 13 & $(2,8)$ \\
\hline$v_{14}$ & Ring $\mathrm{C}=\mathrm{C}$ twist & 358 & $(\mathrm{w}, 22)$ & 353 & $(9,30)$ & 41 & 38 & $(0.3,15)$ & 32 & 35 & $(0.7,14)$ & -9 & -10 & $(2,23)$ \\
\hline \multicolumn{15}{|l|}{ B1 } \\
\hline$v_{15}$ & $=\mathrm{C}-\mathrm{H}$ stretch & 3064 & $(s, 75)$ & 3051 & $(5,44)$ & 0 & -1 & $(4,36)$ & 0 & 0 & $(4,37)$ & 0 & 1 & $(4,25)$ \\
\hline$v_{16}$ & $\mathrm{CH}_{2}$ symmetric stretch & 2912 & $(\mathrm{~s}, 48)$ & 2908 & $(38,65)$ & -24 & -24 & $(24,22)$ & -7 & -10 & $(29,11)$ & -5 & -10 & $(43,10)$ \\
\hline$v_{17}$ & $\mathrm{CH}_{2}$ deformation & 1437 & $(\mathrm{~m}, 4)$ & 1436 & $(10,83)$ & 6 & 6 & $(2,67)$ & 11 & 10 & $(2,61)$ & -4 & -3 & $(5,66)$ \\
\hline$v_{18}$ & $=\mathrm{C}-\mathrm{H}$ in-plane wag & 1353 & $(\mathrm{~m}, 6)$ & 1343 & $(5,10)$ & -5 & -4 & $(2,13)$ & 19 & -34 & $(0.1,8)$ & 13 & 19 & $(1,7)$ \\
\hline$v_{19}$ & $\mathrm{CH}_{2}$ wag & 1284 & $(w, 4)$ & 1268 & $(4,4)$ & 6 & 10 & $(6,8)$ & 29 & -19 & $(4,2)$ & --- & 23 & $(0.1,0.2)$ \\
\hline$v_{20}$ & Ring stretch & 1026 & $(w, 3)$ & 1023 & $(15,30)$ & 31 & 36 & $(12,20)$ & --- & -14 & $(1,22)$ & --- & -19 & $(2,28)$ \\
\hline$v_{21}$ & Ring stretch & 926 & $(\mathrm{~m}, 2)$ & 941 & $(48,6)$ & 0 & -2 & $(0.2,3)$ & --- & -11 & $(10,1)$ & --- & -19 & $(5,0.5)$ \\
\hline$v_{22}$ & Ring angle bend & 797 & $(w, 5)$ & 757 & $(7,8)$ & -12 & -14 & $(0.5,14)$ & -5 & -7 & $(0.4,11)$ & 0 & 2 & $(1,9)$ \\
\hline \multicolumn{15}{|l|}{ B2 } \\
\hline$v_{23}$ & $\mathrm{CH}_{2}$ antisymmetric stretch & 2947 & $(\mathrm{~s}, 80)$ & 2972 & $(20,43)$ & 0 & 1 & $(19,47)$ & -8 & -12 & $(21,36)$ & -27 & -20 & $(6,33)$ \\
\hline$v_{24}$ & $\mathrm{CH}_{2}$ twist & 1122 & $(w, 2)$ & 1143 & $(2,30)$ & 117 & 102 & $(3,18)$ & --- & 43 & $(3,18)$ & --- & 56 & $(5,49)$ \\
\hline$v_{25}$ & $\mathrm{CH}_{2}$ rock & 934 & $(\mathrm{~m}, 4)$ & 934 & $(24,4)$ & 50 & 51 & $(4,16)$ & --- & 97 & $(2,27)$ & 59 & 56 & $(8,6)$ \\
\hline$v_{26}$ & $=\mathrm{C}-\mathrm{H}$ out- of-plane wag & 671 & $(\mathrm{~s}, 11)$ & 668 & $(66,29)$ & 9 & 16 & $(41,27)$ & 15 & 22 & $(27,30)$ & -10 & -4 & $(15,27)$ \\
\hline$v_{27}$ & Ring puckering & --- & $(---,---)$ & 143 & $(1,11)$ & --- & -12 & $(0.3,5)$ & --- & -23 & $(0.7,3)$ & --- & -22 & $(0.6,13)$ \\
\hline$\underline{\mathrm{C}-\mathrm{H} \text { vil }}$ & rations & & & & & & & & & & & & & \\
\hline$v_{\mathrm{CH}}$ & C-H stretch & 2939 & $(s, 67)$ & 2959 & $(56,75)$ & -37 & -37 & $(18,25)$ & -97 & -106 & $(36,54)$ & -82 & -79 & $(29,19)$ \\
\hline$\omega_{\mathrm{CH}}$ & C-H wag (up and down) & 1379 & $(\mathrm{~m}, 7)$ & 1371 & $(9,38)$ & 9 & 5 & $(11,34)$ & -19 & -18 & $(24,46)$ & -24 & -26 & $(18,45)$ \\
\hline$\omega_{\mathrm{CH}}^{\prime}$ & C-H wag (sideways) & 1244 & $(w, 5)$ & 1214 & $(17,19)$ & -48 & 31 & $(3,8)$ & 95 & 9 & $(1,2)$ & -39 & -39 & $(0.9,43)$ \\
\hline $\mathrm{NH}_{2} \mathrm{vib}$ & rations & & & & & & & & & & & & & \\
\hline$v_{\mathrm{a}-\mathrm{NH}_{2}}$ & $\mathrm{NH}_{2}$ antisymmetric stretch & 3391 & $(\mathrm{vw}, 7)$ & 3406 & $(2,28)$ & 12 & 13 & $(1,25)$ & 3 & 8 & $(2,30)$ & 5 & 11 & $(0.9,19)$ \\
\hline$v_{\mathrm{S}-\mathrm{NH}_{2}}$ & $\mathrm{NH}_{2}$ symmetric stretch & 3329 & $(w, 32)$ & 3313 & $(0.4,53)$ & 6 & 13 & $(0.3,50)$ & 6 & 13 & $(0.05,65)$ & 6 & 12 & $(0.1,40)$ \\
\hline$\delta_{\mathrm{NH}_{2}}$ & $\mathrm{NH}_{2}$ deformation & 1623 & $(\mathrm{~m}, 27)$ & $1612^{\mathrm{d}}$ & $(16,58)^{d}$ & 0 & $0^{\mathrm{d}}$ & $(13,73)^{\mathrm{d}}$ & 6 & $6^{\mathrm{d}}$ & $(13,65)^{\mathrm{d}}$ & -6 & -2 & $(23,29)$ \\
\hline$t_{\mathrm{NH}_{2}}$ & $\mathrm{NH}_{2}$ twist & 1109 & $(w, 58)$ & 1119 & $(7,12)$ & 122 & 122 & $(0.7,18)$ & 115 & 104 & $(0.1,24)$ & 8 & 8 & $(0.9,0.2)$ \\
\hline$\omega_{\mathrm{NH}_{2}}$ & $\mathrm{NH}_{2}$ wag & 849 & $(\mathrm{~m}, 4)$ & 891 & $(100,13)$ & -36 & -36 & $(100,6)$ & -18 & -19 & $(100,9)$ & -40 & -41 & $(100,24)$ \\
\hline$\tau_{\mathrm{NH}_{2}}$ & $\mathrm{NH}_{2}$ torsion & 264 & $(\mathrm{~m}, 1)$ & 279 & $(54,11)$ & -7 & -14 & $(32,8)$ & 13 & $22^{\mathrm{e}}$ & $(21,6)$ & -10 & $-14^{\mathrm{e}}$ & $(27,9)$ \\
\hline$\underline{\mathrm{C}-\mathrm{N}}$ vib & rations & & & & & & & & & & & & & \\
\hline$v_{\mathrm{CN}}$ & C-N stretch & 1086 & $(\mathrm{vw}, 2)$ & 1068 & $(2,34)$ & 44 & 54 & $(10,30)$ & 36 & 34 & $(7,22)$ & 0 & 0 & $(1,44)$ \\
\hline$\omega_{\mathrm{CN}}$ & C-N wag & 448 & $(\mathrm{vw}, 6)$ & 429 & $(14,2)$ & 11 & 4 & $(1,9)$ & 44 & 19 & $(10,5)$ & -19 & -19 & $(2,0.9)$ \\
\hline$\omega_{\mathrm{CN}}^{\prime}$ & C-N wag & 366 & $(\mathrm{w}, 8)$ & 379 & $(5,6)$ & -38 & -34 & $(13,12)$ & -12 & -18 & $(8,3)$ & 8 & 6 & $(6,4)$ \\
\hline
\end{tabular}

${ }^{\mathrm{a}}$ Observed frequencies in $\mathrm{cm}^{-1}$.

${ }^{b}$ Relative infrared (IR) and Raman (R) intensities.

'From MP2/cc-pVTZ computations unless indicated.

${ }^{\mathrm{d}} \mathrm{C}=\mathrm{C}$ stretch and $\mathrm{NH}_{2}$ deformation vibrations are coupled.

${ }^{\mathrm{e}}$ Approximate shifts calculated from torsional PEFs from eq. 3. 


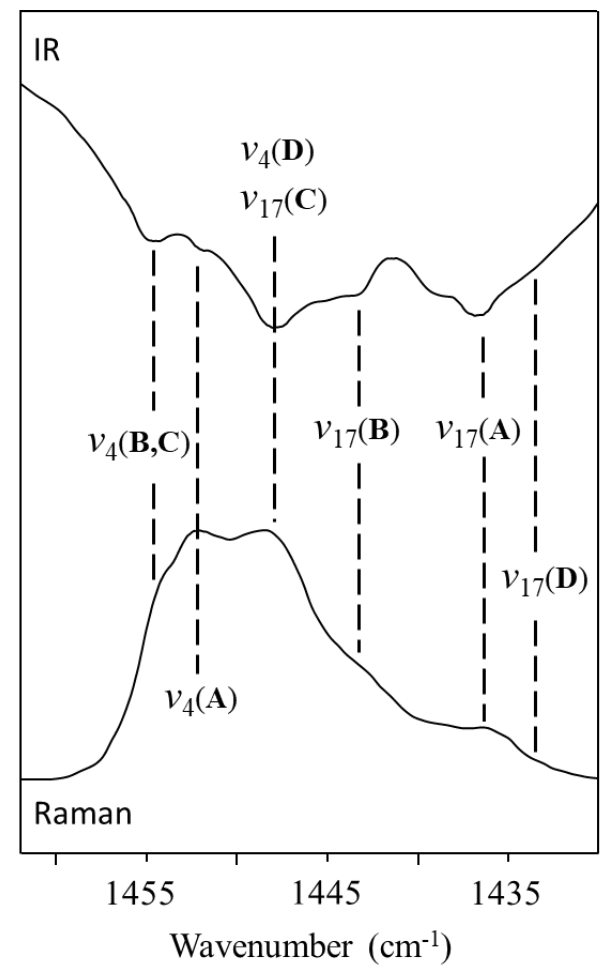

Figure S1. Vibrational spectra of the $\mathrm{CH}_{2}$ deformation vibrations ( $v_{4}$ and $v_{17}$ ).

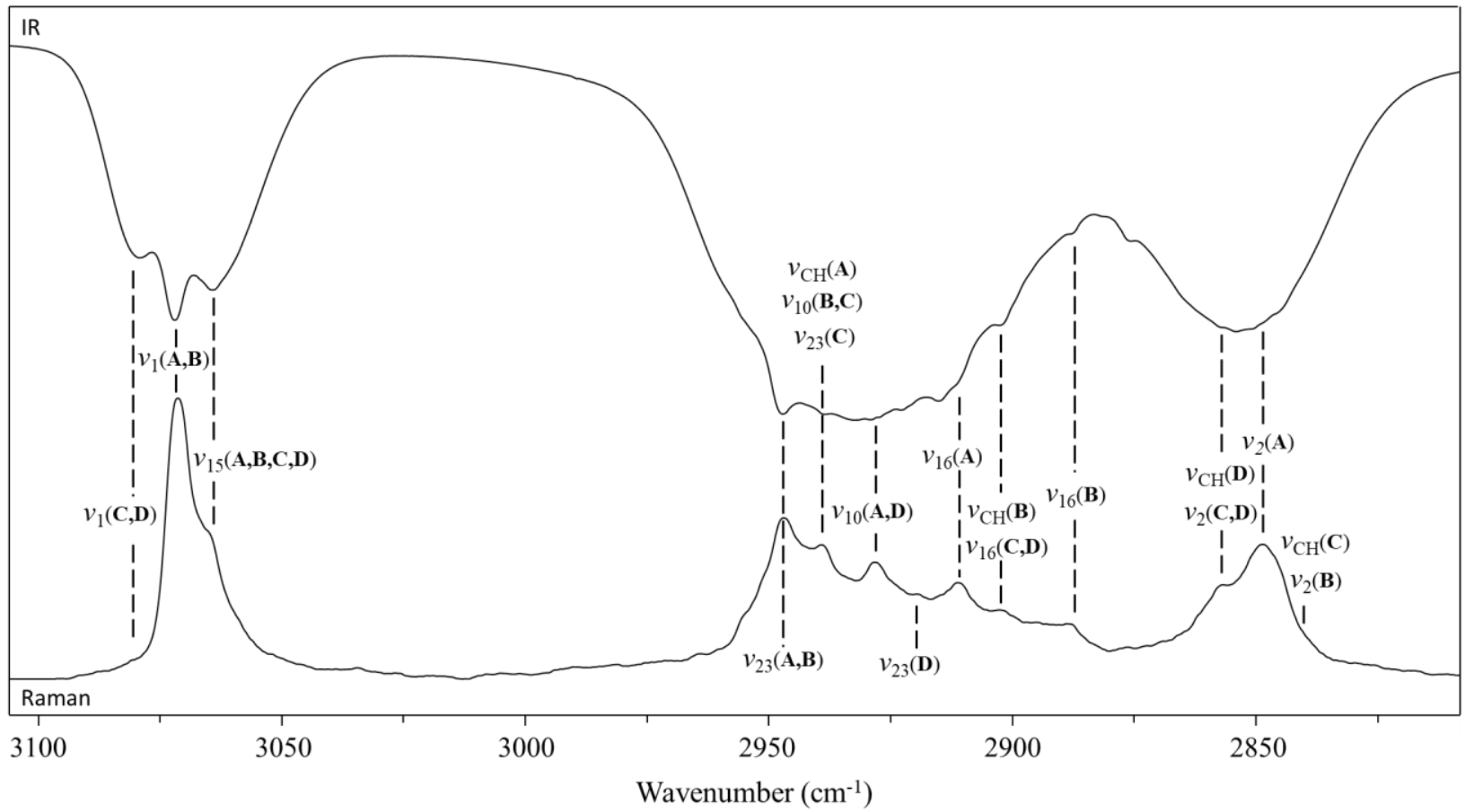

Figure S2. 3CPAM vibrational spectra in the $2800-3100 \mathrm{~cm}^{-1}$ region. 


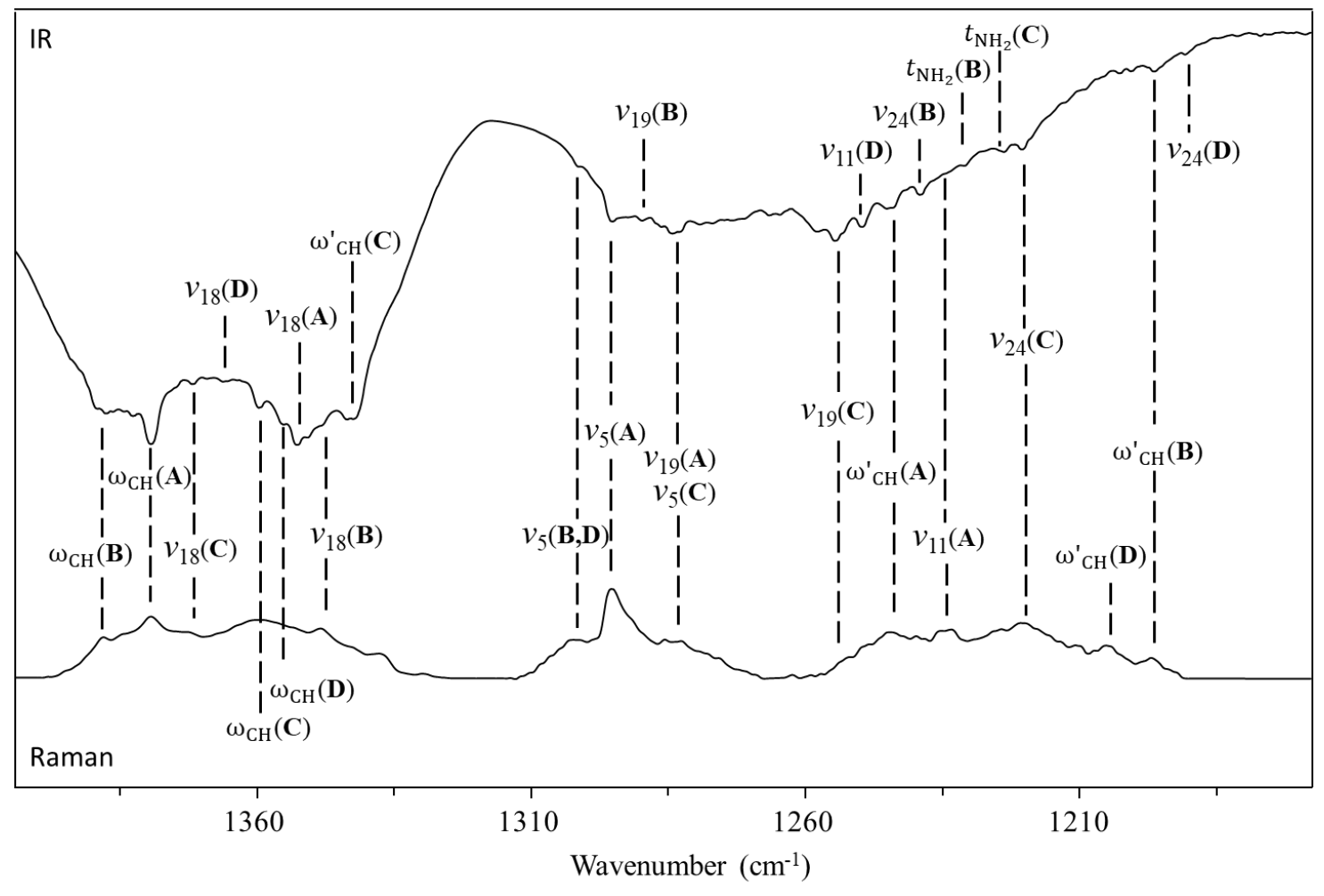

Figure S3. 3CPAM vibrational spectra in the $1180-1450 \mathrm{~cm}^{-1}$ region.

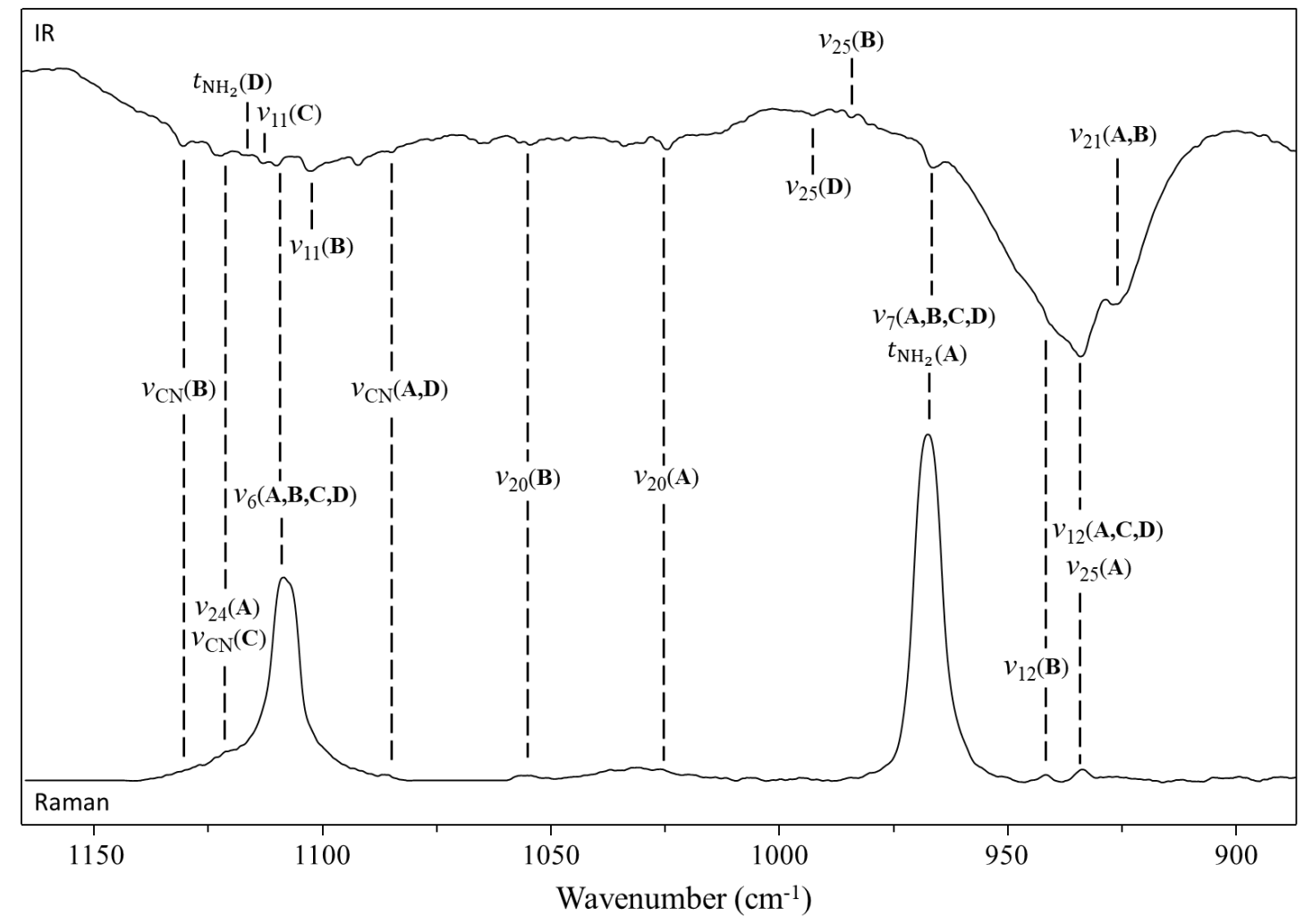

Figure S4. 3CPAM vibrational spectra in the $900-1150 \mathrm{~cm}^{-1}$ region. 


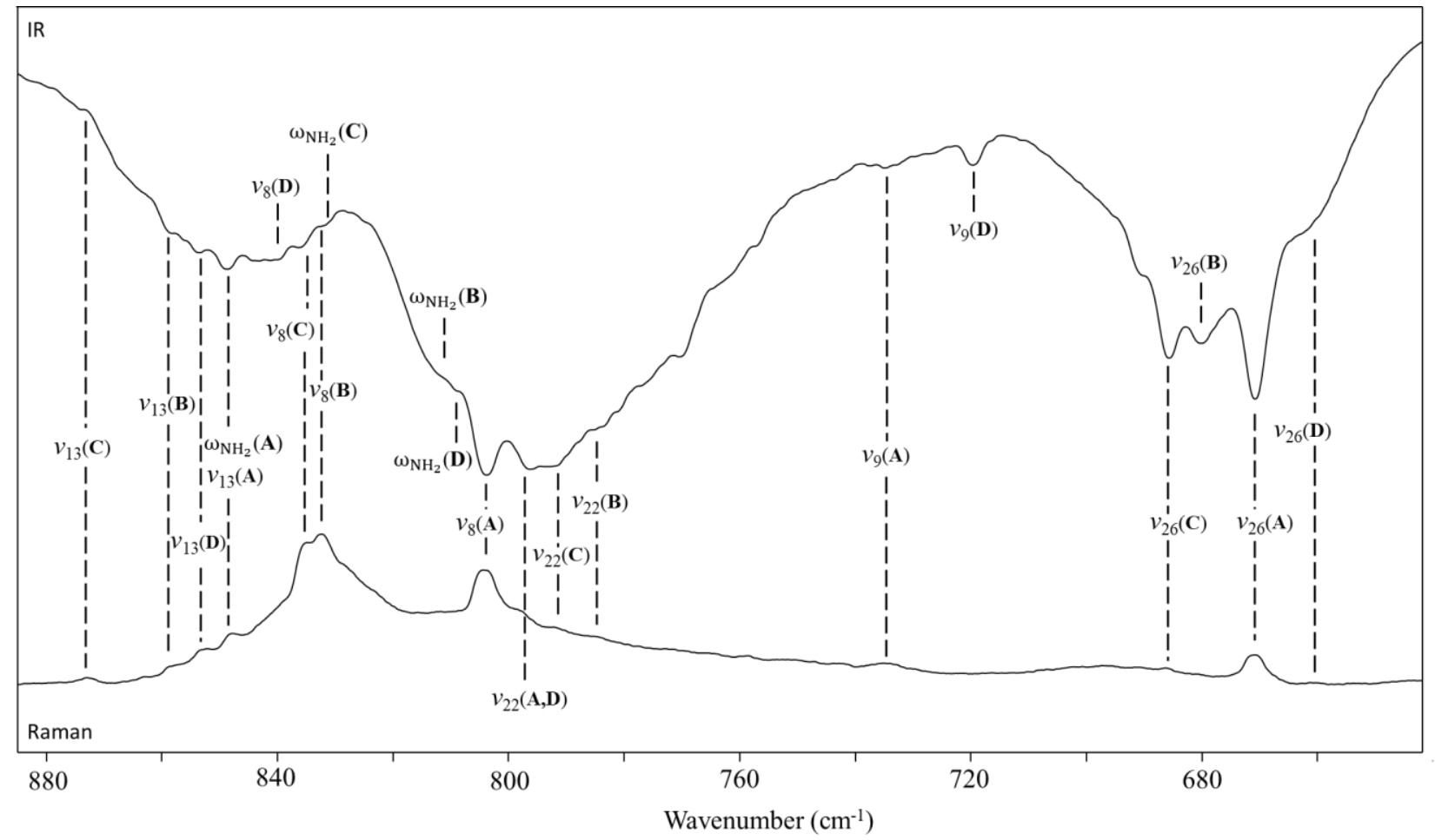

Figure S5. 3CPAM vibrational spectra in the $630-880 \mathrm{~cm}^{-1}$ region.

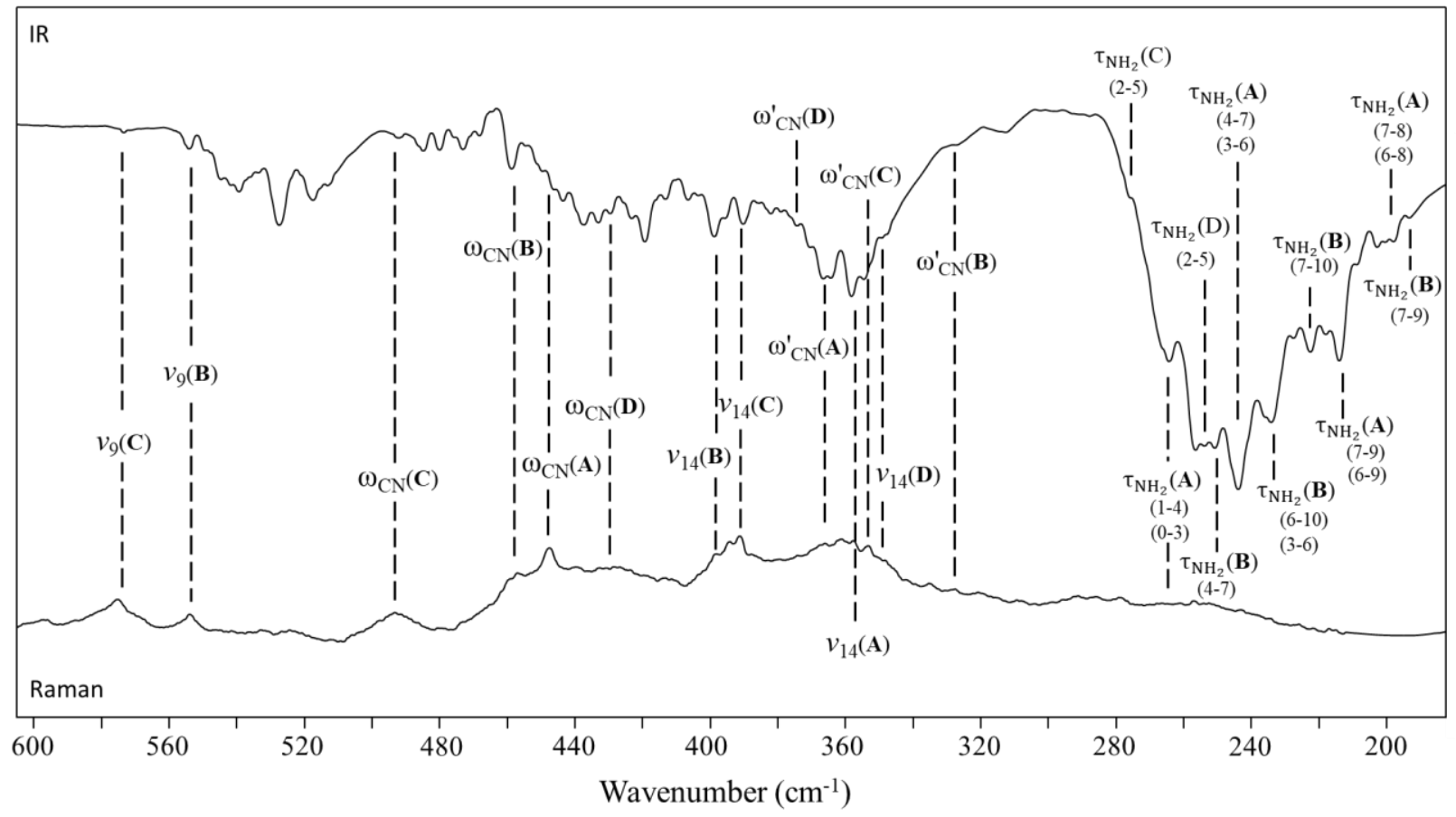

Figure S6. 3CPAM vibrational spectra in the $185-600 \mathrm{~cm}^{-1}$ region. 